\title{
More Than 25 Years of Surgical Treatment of Hydatid Cysts in a Nonendemic Area Using the "Frozen Seal" Method
}

\author{
J. H. M. B. Stoot $\cdot$ C. K. Jongsma • \\ I. Limantoro $\cdot$ O. T. Terpstra $\cdot$ P. J. Breslau
}

Published online: 7 November 2009

(c) The Author(s) 2009. This article is published with open access at Springerlink.com

\begin{abstract}
Background Hydatid disease of the liver remains endemic in the world and is an imported disease in The Netherlands. The aim of this study was to evaluate the treatment and outcome of surgically treated patients for hydatid disease in a single center in The Netherlands.

Methods This retrospective study included 112 consecutive patients surgically treated for hydatid disease between 1981 and 2007. The primary outcome was relapse of the disease. Secondary outcomes were infections, complications, reoperations, length of hospital stay, and mortality.
\end{abstract}

This study was presented to the 8th World Congress of the International Hepato-Pancreatico-Biliary Association in Mumbai, India.

J. H. M. B. Stoot $(\bowtie) \cdot$ I. Limantoro

Department of Surgery, Maastricht University Medical Center,

P. Debeylaan 25, PO Box 5800, 6202AZ Maastricht,

The Netherlands

e-mail: jan@stoot.com

C. K. Jongsma

Department of Surgery, Harbour Hospital, Rotterdam,

The Netherlands

O. T. Terpstra

Department of Surgery, Leiden University Medical Center,

Leiden, The Netherlands

\section{P. J. Breslau}

Department of Surgery, Haga Hospital Location Red Cross,

The Hague, The Netherlands

Present Address:

P. J. Breslau

Medische Specialisten Registratie Commissie (MSRC), Utrecht,

The Netherlands
Results In all cases, echinococcosis was diagnosed by computed tomography or ultrasonography (US). Serology (enzyme-linked immunosorbent assay, immunofluorescence) confirmed the diagnosis in $92.9 \%$. Most of the cysts were seen only in the liver (73.5\%). All cysts were operated on with the frozen seal technique. Relapse of disease was seen in $9(8.0 \%)$ cases. Five $(4.5 \%)$ required surgical treatment at a later stage. Twenty (17.9\%) complications were recorded. Four $(3.6 \%)$ needed radiological drainage and three $(2.7 \%)$ a reoperation. Follow-up was performed with US and/or serology at a mean of 24 months (range 0.5-300 months). All but one complication were seen in the liver-operated group, this proved not to be of statistical significance $(P=0.477)$. Patients with complications stayed significantly longer in hospital than did the patients without complications $(P<0.001)$. No mortality was observed in this study.

Conclusions The present study suggests that the frozen seal method of surgery for hydatid disease is safe and effective. Future studies are needed to prove its position in the treatment of hydatid disease as new developments show promising results.

\section{Introduction}

Hydatid cyst disease, or human cystic echinococcosis, is a zoonotic infection mainly caused by the larval forms of the tapeworm Echinococcus granulosus [1-8]. Echinococcosis occurs worldwide in endemic large sheep-raising areas including Africa, the Mediterranean region of Europe, the Middle East, Asia, South America, Australia, and New Zealand [2, 3, 5-10]. The incidence of hydatid disease in Western industrial nations is relatively low $[6,8,10,11]$. However, the prevalence of the disease has increased in 
Europe and North America due to immigration and traveling [5, 12]. In The Netherlands it is almost always an imported disease $[13,14]$. The most frequent site for cystic lesions is the liver ( $60 \%$ of cases) followed by the lungs (about $20 \%$ of cases). In fact, infection can affect all tissues in the human body [1, 3, 6-8, 10, 11, 15].

There are three treatment options for hepatic cystic echinococcosis: chemotherapy, surgery, and percutaneous drainage (puncture, aspiration, injection, reaspiration-also known as PAIR) or a combination of these therapies [6-8]. Although PAIR has been investigated by several groups, there is still no consensus about the best treatment. Surgery may vary from conservative (cystectomy) to radical (complete open resection) to laparoscopic techniques. The debate on the best surgical approach is still ongoing, whether it is conservative surgery or radical surgery in which the cyst is totally removed including the pericyst by total pericystectomy or partial hepatectomy [16].

In an area where hydatid disease is not endemic and therefore not a routine treatment, it is of major importance to perform a safe, reproducible technique with a low complication rate. The frozen seal method as surgical treatment for hydatid disease, described by Saidi and Nazarian during the 1970s, has been used in the Harbour Hospital since 1981 [17]. The aim of this study was to investigate the results of the frozen seal method as treatment of echinococcosis in a specialized center in The Netherlands over the past 25 years.

\section{Methods}

This study included all consecutive surgically treated patients in the Harbour Hospital in Rotterdam, The Netherlands, between 1981 and 2007. All medical records were reviewed concerning the following parameters: age, sex, country of origin, presentation of symptoms, location and type of cysts diagnosed by radiologic imaging, treatment, hospital stay, and outcomes. Radiologic workup included conventional radiology, ultrasonography (US), and computed tomography (CT). Laboratory tests and serology were performed to confirm the diagnosis. Enzyme-linked immunosorbent assay (ELISA), immunoelectrophoresis (IE), and in most cases the complement binding reaction (CBR) were used as serologic testing for diagnosis and follow-up.

Primary outcome was relapse of disease. Secondary outcomes were infections, complications, spilling of hydatid cyst content, reoperations, length of stay, and mortality.

An echinococcal cyst was diagnosed if any two of the features were present: (1) typical sonographic and CT appearance demonstrating a multilocular cystic lesion with daughter cysts, calcification of the cysts walls, or both; (2) positive indirect hemagglutination test or ELISA for echinococcal infection; and (3) histology demonstrating a parasite-derived laminated membrane with or without viable scolices $[4,11,18]$. Indications for surgery were the presence of pain, jaundice, fever, dyspnea, or cardiac decompensation. The World Health Organization (WHO) indications for surgery were respected in all cases: large liver cysts with multiple daughter cysts, infected cysts, and cysts communicating with the biliary tree. Moreover, surgery was considered indicated in the presence of cysts in the lung, heart, and other organs [11, 15].

Prior to the operation in some cases ( $n=28,25.0 \%)$ albendazole (400 $\mathrm{mg}$ twice a day) was administered and continued 3 months after surgery. Before 1988, mebendazole was used in selected cases $(n=19,17.0 \%)$. From 1990, albendazole was given depending on the patient's clinical situation (severe pain, fever, other clinical signs of echinococcosis infection). From 1995, the regimen was standardized, and almost all patients received preoperative drug treatment (14/15 patients), One patient received Eskazole (albendazole).

\section{Surgical technique}

In this study, the frozen seal technique was used for open surgery in all cases. For hydatid cysts in the lung or myocardium, the thorax was opened via a median sternotomy incision. For liver cysts, the abdomen was exposed through a bilateral subcostal incision, abdominal viscera were examined for potential sites of dissemination, and the liver was mobilized. Using this technique, a $7 \mathrm{~cm}$ long metal cone with a large $(8 \mathrm{~cm}$ diameter) upper and $4 \mathrm{~cm}$ lower aperture was used. It has fine tubing soldered to the rim of the lower opening. A refrigerant is circulated via the inlet of the tubing. In this setting, Freon was used in all cases as refrigerant. The ring-shaped tubing of the cone was frozen onto the cyst on the surface of the liver (or lung), producing a watertight frozen seal. The contents of the cysts could then be evacuated through the cone without risk of uncontrolled rupture or spilling [13, 17, 19]. Normally, the fluid is crystal clear. If the fluid was turbid, a communication with bile ducts or secondary infection was suspected, and material for bacteriologic examination was obtained (and, consequently, antibiotic treatment was started).

Then the membrane was excised completely and the residual space was meticulously inspected for biliary communication. If there was any suspicion of biliary communication, fistulas were closed with absorbable sutures. If no communication was present, the residual cavity was flushed for 2 min with a freshly prepared $0.5 \%$ silver nitrate solution to kill any remaining scolices. The 
use of this scolicidal agent was based on the evidence provided by Saidi and Nazarian [17, 20]. Silver nitrate $0.5 \%$ solution proved to be a suitable alternative for formalin in a mouse model with standardized intraperitoneal inoculation techniques. It effectively sterilized scolices within 2 min and, more importantly, was completely nontoxic to other tissues. Clinical application of silver nitrate $0.5 \%$ led to effective treatment without any local or systemic effect of the silver nitrate washout in more than 55 surgically treated patients with liver and lung hydatid cysts [20].

After the use of silver nitrate solution, crypts or recesses were carefully checked for residual cyst material. If present, it was removed, and a second treatment with silver nitrate solution was administered.

Again, the residual cavity was meticulously inspected for biliary communication. Any visible fistulas were sutured with absorbable sutures. If the residual space communicated with bile ducts or bronchus, any contact between the silver nitrate solution and these structures was avoided either by compressing the liver or increasing the end-expiratory pressure of lung ventilation. No residual space should be present after this procedure as it probably enhances the possibility of infection. Therefore, the cavity was filled with hypertonic saline and closed with nonabsorbable sutures or, in selected cases, obliteration with greater omentum was performed.

If appropriate, cavities were drained with $18 \mathrm{~F}$ vacuum tubes, and an additional subhepatic drain was placed in selected cases to control any possible biliary leakage. Drains were removed on the fifth to seventh postoperative day. Patients who required prolonged drainage were considered to have biliary fistulas. For patients with symptoms of an obstructed or communicating common bile duct (CBD), intraoperative cholangiography and choledochotomy with T-tube drainage were performed. In selected cases, endoscopic retrograde cholangiopancreatography (ERCP) was performed to drain the CBD.

\section{Follow-up}

Complications were defined as "every unwanted development in the illness of the patient or in the treatment of the patient's illness that occurs in the clinic" [21]. Complications were classified according to the recent grading system of Dindo et al. (Table 1) [22]. Spilling was considered an error in technique that could lead to a complication (infection) or relapse of disease.

After discharge, all patients were followed periodically at the first, third, sixth, and twelfth postoperative month during the first year and at least once a year thereafter. Seamen were unable to be followed long term. The followup procedure included physical examination, serologic tests, US of the abdomen, and CT scanning in particular cases. Relapse or recurrence of disease was defined as the appearance of new extrahepatic disease resulting from procedure-related spilling or reappearance of live cysts at the site of a previously treated cyst $[7,23]$.

Table 1 Classification of surgical complications

\begin{tabular}{|c|c|}
\hline Grade $^{1}$ & Definition \\
\hline \multirow[t]{2}{*}{ I } & $\begin{array}{l}\text { Any deviation from the normal postoperative course without the need for pharmacologic treatment or surgical, endoscopic, and } \\
\text { radiologic interventions }\end{array}$ \\
\hline & $\begin{array}{l}\text { Allowed therapeutic regimens are drugs as antiemetics, antipyretics, analgesics, diuretics, electrolytes, and physiotherapy. This grade } \\
\text { also includes wound infections opened at the bedside }\end{array}$ \\
\hline \multirow[t]{2}{*}{ II } & Requiring pharmacologic treatment with drugs other than such allowed for grade I complications \\
\hline & Blood transfusions and total parenteral nutrition are also included \\
\hline III & Requiring surgical, endoscopic, or radiologic intervention \\
\hline IIIa & Intervention not under general anesthesia \\
\hline IIIb & Intervention under general anesthesia \\
\hline IV & Life-threatening complication (including CNS complications) ${ }^{2}$ requiring IC/ICU management \\
\hline IVa & Single organ dysfunction (including dialysis) \\
\hline $\mathrm{IVb}$ & Multiorgan dysfunction \\
\hline $\mathrm{V}$ & Death of a patient \\
\hline
\end{tabular}

From Dindo et al. [22]

$C N S$ central nervous system, $I C$ intermediate care, $I C U$ intensive care unit

1 The suffix "d" (for "disability") is added to the respective complication grade if the patient suffers from a complication at the time of discharge. It indicates the need for follow-up to evaluate the complication fully

${ }^{2}$ Brain hemorrhage, ischemic stroke, subarachnoidal bleeding but excluding transient ischemic attacks 
Statistical analysis

Categoric variables were presented as numbers (percentages) and continuous variables by the median and range. Complications were analyzed using Fisher's exact test. Hospital stay was analyzed using the Mann-Whitney $U$-test. The analyses did not account for missing data. A value of $P<0.05$ was considered to be statistically significant. Data were analyzed using SPSS version 13.0 for Windows (SPSS, Chicago, IL, USA).

\section{Results}

The study describes a total of 112 consecutive patients who underwent surgery for hydatid disease during the years 1981-2007 at Harbour Hospital, Rotterdam, The Netherlands. All but five patients were from abroad, either immigrants or seamen. Demographic data are presented in Table 2. At clinical presentation, the complaints consisted mainly of abdominal pain $[n=87(77.7 \%)]$, abdominal mass $[n=43(38.4 \%)]$, fever $[n=20(17.9 \%)]$, and urticaria $[n=11(9.8 \%)]$ or a combination of these symptoms. Also, acute abdominal complaints due to rupture $[n=3$ (2.7\%)], cardiac decompensation [ $n=2(1.8 \%)]$, anaphylaxis $[n=1(0.9 \%)]$ and jaundice $[n=8(7.1 \%)]$ were seen at presentation. In all cases, echinococcosis was diagnosed by US and CT scanning. Serology [ELISA, immunofluorescence (IMF)] confirmed the diagnosis in $92.9 \%$ of the cases. Liver function tests were abnormal only when the hepatic ducts were involved.

Table 2 Demographic data from patients treated for hydatid disease in the Harbour Hospital: 1981-2007

\begin{tabular}{ll}
\hline Age (median, range) & $38(7-70)$ years \\
Sex (M:F) & $52: 60$ \\
No. of patients, by country of origin & \\
Armenia & 1 \\
India & 1 \\
Italy & 2 \\
Iraq & 1 \\
Lebanon & 1 \\
Libya & 1 \\
Morocco & 37 \\
The Netherlands & 5 \\
Spain & 3 \\
Sudan & 1 \\
Turkey & 52 \\
Uruguay & 1 \\
Unknown & 4 \\
Yugoslavia & 2 \\
Total & 112 \\
\hline
\end{tabular}

Most of the cysts were seen only in the liver, although other, more rare locations were found including the myocardium and the adductores muscles in the right leg. The locations are given in Table 3 together with the number of complications per organ. Surgery was performed in all cases $[n=112]$ with the frozen seal method, and more than 196 cysts were operated on in the liver, lungs, and abdomen. Primary and secondary outcomes were scored in the clinic and after discharge during a median follow-up of 24 months (0.5-300 months) (Table 4). Twelve patients $(10.7 \%)$ were lost to follow-up. Relapse of disease was observed in nine patients (8.0\%) during a median follow-up period of 33 months (10-300 months). In this group with recurrence $(n=9), 6$ patients were initially treated before and after surgery with medication (mebendazole $n=2$, abendazole $n=4$, no medication $n=3$ ). Regarding further treatment, four patients $(3.6 \%)$ needed five reoperations $(4.5 \%)$ at a later stage. The remaining five patients were treated successfully with albendazole $(400 \mathrm{mg}$ twice a day).

Grade I complications $(n=2)$ consisted of two wound infections. The moderate complications (grade II, $n=10$ ) were two biliary fistulas, two pneumonias, two urinary tract infections, and four infections of the remnant cavity. More severe complications (grade III) with interventions consisted of fascia dehiscence, pleural effusion, and cavity infections and/or abscesses that required radiological drainage $(n=4)$ or relaparotomy $(n=3)$. One grade IV complication, acute respiratory distress syndrome (ARDS), occurred after percutaneous treatment of a hydatid cyst in the liver in segment 8 due to perforation and rupture to the lung. No complications or abnormal liver function tests were noted related to the use of silver nitrate $0.5 \%$ solution. No mortality was observed in this study.

All but one of the complications were observed in the liver-operated group, which proved not to be of statistical significance $(P=0.477)$. Even after dividing the hydatid locations in only two groups-liver (19/99 patients, or $19.2 \%$ complication rate) and other organs $(1 / 13$, or $7.7 \%$ complication rate)-no significant difference was found $(P=0.457)$. Also, no significant difference was seen when the grading of complications was analyzed $(P=0.881)$. However, if a complication occurred, these patients stayed significantly longer in hospital than the patients without complications $(P<0.001)$. The median stay was 20.5 days (10-156 days) in the group with complications $(n=20)$ versus 12 days (2-52 days) in those without complications $(n=87)$.

Of the 112 consecutive patients, 3 were treated with a percutaneous technique prior to surgical treatment. This was decided in a multidisciplinary meeting prior to the intervention, and it was considered suitable for treatment. The first patient needed a thoracoabdominal exploration 
Table 3 Number, location, and largest diameter of hydatid cysts from patients surgically treated in the Harbour Hospital: 19812007

In some records the diameter and/or the numbers of cysts were not reported but were regarded as multiple. These are not reported in this table*

\begin{tabular}{lcc}
\hline Organ & No. of cysts & $\begin{array}{c}\text { Complications } \\
\text { (no. of patients) }\end{array}$ \\
\hline Liver* (no. of patients) & $144(73.5 \%)$ & $19(95.0 \%)$ \\
Grade I $(n=2)$ & & \\
Grade II $(n=10)$ & & \\
Grade IIIa $(n=3$ & & $1(5.0 \%)$ \\
Grade IIIb $(n=3)$ & $26(13.3 \%)$ & \\
Grade IV $(n=1)$ & $5(2.6 \%)$ & \\
Lung* & $4(2.0 \%)$ & \\
Myocardium* (grade IIIa) $(n=1$ patient) & $3(1.5 \%)$ & \\
Spleen & & \\
Bladder & $6(3.1 \%)$ & \\
Other & $1(0.5 \%)$ & \\
m. psoas & $2(1.0 \%)$ & \\
m. adductores & $2(1.0 \%)$ & \\
Mesocolon & $3(1.5 \%)$ & \\
Retroperitoneum & 196 & \\
Skin & $8.6(2-28)$ & \\
Total patients & & \\
Diameter $(\mathrm{cm})$, mean and range & & \\
\hline
\end{tabular}

Table 4 Primary and secondary outcomes of all 112 consecutive patients surgically treated for hydatid disease in the Harbour Hospital: 1981-2007

\begin{tabular}{lc}
\hline Primary outcome & \\
Relapse of disease & $9(8.0 \%)$ \\
Intervention (reoperation) & $5(4.5 \%)$ \\
Secondary outcome & \\
Complications & \\
Grade I & $2(1.8 \%)$ \\
Grade II & $10(8.9 \%)$ \\
Grade IIIa & $4(3.6 \%)$ \\
Grade IIIb & $3(2.7 \%)$ \\
Grade IV(d) & $1(0.9 \%)$ \\
Grade V (death) & 0 \\
Reoperation & $3(2.7 \%)$ \\
Length of hospital stay (mean, range) & $14(2-156)$ days \\
\hline
\end{tabular}

Results are the number and percent of patients unless otherwise indicated

because biliary pleuritis persisted after PAIR and required surgical treatment. Another patient was treated by PAIR for a hydatid cyst in the liver and was operated on with the frozen seal technique for the spleen without complication. The third patient was the previously described patient with ARDS (grade IVd complication).

Spilling of cyst content was reported in four cases, without complications or recurrence in these patients during follow-up of 22.5 months (range 5-39 months). Two ERCPs were performed prior to the liver operation. An
ERCP was done in the first patient to analyze the cause of the abnormal liver function tests. This patient was admitted in 1981 to the Department of Internal Medicine. After CT scanning, he was referred to the Department of Surgery to be treated surgically. The second ERCP was performed 2 years later in a patient to analyze the cause of persistent high bilirubin levels in the serum. The sonography showed an enlarged biliary tree and a cystic process in the right liver lobe. A parasitic infection was suspected at first, but serology was negative for echinococcosis. ERCP showed a stone in the common bile duct (CBD). Four days later, cholecystectomy with CBD exploration was planned. However, the "stone" seen by ERCP proved to be a large daughter cyst and was removed. The large hydatid cyst in the liver was also treated, and T-tube drainage was performed. Postoperative medical treatment with mebendazole was started, and the patient was discharged in good condition 1 month later.

\section{Discussion}

The present study showed that the use of a previously described frozen seal technique is safe and effective for the surgical treatment of hydatid disease in a nonendemic area. No mortality was seen in more than 25 years of surgically treated echinococcosis, and the recurrence rate and morbidity were low. In fact, the results can be compared favourably to the recent meta-analysis where the clinical outcomes of 769 patients with hepatic echinococcosis 
treated with the PAIR technique were compared with 952 era-matched historical controls undergoing surgical intervention [8]. In this meta-analysis of 14 studies on surgical treatment in endemic areas, minor and major complication rates of $33.0 \%$ and $25.1 \%$, respectively, were reported. The mortality rate was $0.7 \%(n=7)$ among the surgically treated patients. In a more recent single-center study of this matter with 171 patients, a mortality rate of $1 \%$ was reported for 10 years of open surgical treatment [7]. For this group of open surgery, the investigators observed a morbidity rate of $28 \%$ and a recurrence rate of $16.2 \%$.

A drawback of the present study may be the retrospective setting, which could account for a bias for the comparison with reports in the literature. However, most of the studies in the field of surgical treatment of hydatid disease are of the same retrospective design [2, 7, 24-31].

There are different opinions concerning the optimal treatment for patients with hepatic echinococcal disease. The goal of treatment of hydatid disease is to eliminate the parasite completely and thereby prevent (or treat) complications including secondary infection, rupture of the cyst, anaphylactic reaction, pressure on adjacent structures, and penetration into the biliary tract. It is also important to prevent recurrent disease as it is known that there is a considerable rate of recurrence, possibly caused by intraoperative spilling $[5,7,11,32]$.

In theory, there are three treatment options for hepatic cystic echinococcosis: chemotherapy, surgery, and percutaneous drainage or a combination of these therapies [7, 8]. However, the use of chemotherapeutic agents alone, such as mebendazole or albendazole, is controversial because of their limited efficacy. These antiparasitic drugs are often administered as adjuvant therapy during surgery or percutaneous treatment. In selected cases, they can be the primary approach when surgery is not feasible or is unsafe [8, 10, 32].

Percutaneous drainage is another method for treating hepatic hydatid disease. The PAIR technique consists of several sequential steps: After percutaneous aspiration of the hydatid cyst under US or CT guidance, a protoscolicidal agent ( $20 \%$ sodium chloride solution or $95 \%$ ethanol) is injected into the cyst cavity; the final step is reaspiration of the cyst contents. The advantage of PAIR is that it is less invasive [5, 32]. In the case of uncomplicated hepatic hydatid cysts, percutaneous drainage in combination with albendazole therapy is an effective, safe alternative to surgery and requires a shorter hospital stay [2, 3, 7, 10, 31, $33,34]$. In this study, only three patients were treated with PAIR, with two complicated procedures, and these patients needed surgery thereafter.

The third method for managing cystic echinococcosis of the liver is surgery. The surgical approach remains the cornerstone of the treatment for hydatid disease $[1,3,7,10$,
$15,31,32,35]$. Some suggest that a combination of surgery and antiparasitic agents is the preferred treatment as it is associated with low morbidity, mortality, and recurrences rates $[10,36]$. Surgery of liver hydatid cysts is intended to remove the parasite completely by a procedure that includes safe, complete exposure of the cyst, safe decompression of the cyst, safe evacuation of the cyst contents, sterilization of the cyst, treatment of biliary complications, and management of the remaining cyst cavity. Surgeons must also try to prevent intraoperative spilling and to save healthy issue $[1,5,6,11,36]$.

The WHO has published a list of indications for surgical treatment: large liver cysts with multiple daughter cysts; single superficially situated liver cysts that may rupture spontaneously or as the result of trauma; infected cysts; cysts communicating with the biliary tree and/or exerting pressure on adjacent vital organsl and cysts in the lung, brain, kidney, bones, and other organs [11, 15].

Important complications following surgery for hydatid liver echinococcosis are recurrences, with a reported incidence of $1.1 \%$ to $25.0 \%$, which may be caused by spilling the cyst's contents during a previous operation $[4,25,26$, $29,31,32,37]$. In the present study, only four cases of intra-perative spilling were reported $(3.6 \%)$, without any recurrence in almost 2 years of median follow-up.

Surgical interventions can be divided into conservative, radical, and laparoscopic procedures. Conservative, tissuesparing techniques consist of evacuating the cyst and leaving the pericyst in situ, whereas radical procedures remove both the cyst and the pericyst. The most common conservative techniques include simple tube drainage, marsupialization, capitonnage, deroofing, partial cystectomy, and open or closed total cystectomy with or without omentoplasty. Conservative operations have good results regarding blood loss and length of hospital stay [36].

Radical approaches consist of total pericystectomy or liver resection (hemihepatectomy or lobectomy) in which the cyst content and the entire pericystic membrane are removed [1, 2, 4-6, 11]. Radical resection means extensive dissection, leading to a large wound area with a possibly increased risk of bleeding or bile leaks [2, 33, 36]. This technique has also advantages for the treatment of hydatid disease. It is suggested to be a safe, highly effective technique with lower mortality, morbidity, and recurrence rates than for conservative surgery [5, 7, 35]. This can be partially explained by the fact that, compared to endocystectomy, excision of the cyst and pericyst minimizes the risk that daughter cysts persist in situ [10, 36].

Recurrences are often related to spillage of the cyst content [7, 33]. Therefore, Saidi and Nazarian [17] developed a method to prevent spillage of the cyst content. They devised a cone, which simplifies disinfection of the cyst cavity, as used in this study $[11,13,17,19]$. This procedure 
starts with mobilization of the liver so the cone can be centered on the apex of the cyst. After opening the cyst through the cone, the parasite is sucked off entirely, followed by disinfection of the remaining cavity $[11,13,17$, 19].

The laparoscopic approach has a growing role in the treatment of hepatic hydatid cysts [2, 7, 32, 38]. Current data suggest that this minimally invasive surgical technique is feasible, safe, and effective [1, 2, 7, 39-44]. Unfortunately, randomized controlled trials are still lacking, which results in continuing debate about the optimal operation technique for hydatid disease [2, 4, 11, 16, 36]. It is of major importance to select the appropriate therapy carefully, remembering the benign nature of this cystic disease [32]. Careful patient selection is based on the size, number, and location of the cysts, the patient's characteristics, and the experience of the clinician $[4,10]$.

\section{Conclusions}

The frozen seal method of surgery from the past century is still safe and effective in the new millennium. It is especially useful in centers in nonendemic areas to facilitate working with a reproducible technique and, more importantly, to obtain high efficacy and low morbidity rates. Future studies are needed to prove its position in the treatment of hydatid disease as new developments show promising results.

Acknowledgments We thank the surgical staff and Mrs. M.E.I. van Nieuwland, Department of Surgery, Harbour Hospital for the cooperation and kind assistance with this study. We also thank Prof. Dr. C.H.C. Dejong for critically reading this manuscript.

Open Access This article is distributed under the terms of the Creative Commons Attribution Noncommercial License which permits any noncommercial use, distribution, and reproduction in any medium, provided the original author(s) and source are credited.

\section{References}

1. Dervenis C, Delis S, Avgerinos C et al (2005) Changing concepts in the management of liver hydatid disease. J Gastrointest Surg 9:869-877

2. Dziri C, Haouet K, Fingerhut A (2004) Treatment of hydatid cyst of the liver: where is the evidence? World J Surg 28:731-736

3. Khuroo MS, Wani NA, Javid G et al (1997) Percutaneous drainage compared with surgery for hepatic hydatid cysts. N Engl J Med 337:881-887

4. Menezes da Silva A (2003) Hydatid cyst of the liver: criteria for the selection of appropriate treatment. Acta Trop 85:237-242

5. Sayek I, Tirnaksiz MB, Dogan R (2004) Cystic hydatid disease: current trends in diagnosis and management. Surg Today 34 : 987-996
6. Smego RA Jr, Sebanego P (2005) Treatment options for hepatic cystic echinococcosis. Int J Infect Dis 9:69-76

7. Yagci G, Ustunsoz B, Kaymakcioglu N et al (2005) Results of surgical, laparoscopic, and percutaneous treatment for hydatid disease of the liver: 10 years experience with 355 patients. World J Surg 29:1670-1679

8. Smego RA Jr, Bhatti S, Khaliq AA et al (2003) Percutaneous aspiration-injection-reaspiration drainage plus albendazole or mebendazole for hepatic cystic echinococcosis: a meta-analysis. Clin Infect Dis 37:1073-1083

9. Ammann RW, Eckert J (1996) Cestodes: Echinococcus. Gastroenterol Clin North Am 25:655-689

10. Gourgiotis S, Stratopoulos C, Moustafellos P et al (2007) Surgical techniques and treatment for hepatic hydatid cysts. Surg Today 37:389-395

11. Buttenschoen K, Carli BD (2003) Echinococcus granulosus infection: the challenge of surgical treatment. Langenbecks Arch Surg 388:218-230

12. Khuroo MS, Dar MY, Yattoo GN et al (1993) Percutaneous drainage versus albendazole therapy in hepatic hydatidosis: a prospective, randomized study. Gastroenterology 104:1452-1459

13. Jongsma CK, Stuiver PC (1984) The surgical treatment of echinococcosis using a freezing method. Ned Tijdschr Geneeskd 128:391-393

14. Schipper HG, Kager PA (1997) Diagnosis and treatment of unilocular hydatid disease (Echinococcus granulosus infection). Ned Tijdschr Geneeskd 141:984-989

15. Anonymous (1996) Guidelines for treatment of cystic and alveolar echinococcosis in humans: WHO Informal Working Group on Echinococcosis. Bull World Health Org 74:231-242

16. Franciosi CM, Romano F, Porta G et al (2002) Surgical treatment of hydatid disease of the liver: an experience from outside the endemic area. Chir Ital 54:667-672

17. Saidi F, Nazarian I (1971) Surgical treatment of hydatid cysts by freezing of cyst wall and instillation of 0.5 percent silver nitrate solution. N Engl J Med 284:1346-1350

18. Regev A, Reddy KR, Berho M et al (2001) Large cystic lesions of the liver in adults: a 15-year experience in a tertiary center. J Am Coll Surg 193:5-36

19. Jongsma CK (1984) The frozen seal technique in the surgical treatment of hydatid disease in The Netherlands. Acta Leiden $52: 41-44$

20. Saidi F (1977) A new approach to the surgical treatment of hydatid cyst. Ann R Coll Surg Engl 59:115-118

21. Vrancken Peeters MP, Vrancken Peeters MJ, Corion LU et al (2005) Quality control of colorectal surgery with an extensive complication registration system. Dig Surg 22:168-173

22. Dindo D, Demartines N, Clavien PA (2004) Classification of surgical complications: a new proposal with evaluation in a cohort of 6336 patients and results of a survey. Ann Surg 240:205-213

23. Sielaff TD, Taylor B, Langer B (2001) Recurrence of hydatid disease. World J Surg 25:83-86

24. Akcan A, Akyildiz H, Artis T et al (2007) Peritoneal perforation of liver hydatid cysts: clinical presentation, predisposing factors, and surgical outcome. World J Surg 31:1284-1291

25. Alfieri S, Doglietto GB, Pacelli F et al (1997) Radical surgery for liver hydatid disease: a study of 89 consecutive patients. Hepatogastroenterology 44:496-500

26. Balik AA, Basoglu M, Celebi F et al (1999) Surgical treatment of hydatid disease of the liver: review of 304 cases. Arch Surg 134:166-169

27. Chautems R, Buhler LH, Gold B et al (2005) Surgical management and long-term outcome of complicated liver hydatid cysts caused by Echinococcus granulosus. Surgery 137:312-316 
28. Karavias DD, Vagianos CE, Kakkos SK et al (1996) Peritoneal echinococcosis. World J Surg 20:337-340

29. Magistrelli P, Masetti R, Coppola R et al (1991) Surgical treatment of hydatid disease of the liver: a 20-year experience. Arch Surg 126:518-522

30. Sayek I, Yalin R, Sanac Y (1980) Surgical treatment of hydatid disease of the liver. Arch Surg 115:847-850

31. Yorganci K, Sayek I (2002) Surgical treatment of hydatid cysts of the liver in the era of percutaneous treatment. Am J Surg 184:63-69

32. Voros D, Katsarelias D, Polymeneas G et al (2007) Treatment of hydatid liver disease. Surg Infect (Larchmt) 8:621-627

33. Kayaalp C, Sengul N, Akoglu M (2002) Importance of cyst content in hydatid liver surgery. Arch Surg 137:159-163

34. Ustunsoz B, Akhan O, Kamiloglu MA et al (1999) Percutaneous treatment of hydatid cysts of the liver: long-term results. AJR Am J Roentgenol 172:91-96

35. Alonso CO, Moreno GE, Loinaz SC et al (2001) Results of 22 years of experience in radical surgical treatment of hepatic hydatid cysts. Hepatogastroenterology 48:235-243

36. Mueller L, Broering DC, Vashist Y et al (2003) A retrospective study comparing the different surgical procedures for the treatment of hydatid disease of the liver. Dig Surg 20:279-284

37. Vagianos CE, Karavias DD, Kakkos SK et al (1995) Conservative surgery in the treatment of hepatic hydatidosis. Eur J Surg $161: 415-420$
38. Cirenei A, Bertoldi I (2001) Evolution of surgery for liver hydatidosis from 1950 to today: analysis of a personal experience. World J Surg 25:87-92

39. Altinli E, Saribeyoglu K, Pekmezci S et al (2002) An effective omentoplasty technique in laparoscopic surgery for hydatid disease of the liver. JSLS 6:323-326

40. Bickel A, Loberant N, Singer-Jordan J et al (2001) The laparoscopic approach to abdominal hydatid cysts: a prospective nonselective study using the isolated hypobaric technique. Arch Surg 136:789-795

41. Chowbey PK, Shah S, Khullar R et al (2003) Minimal access surgery for hydatid cyst disease: laparoscopic, thoracoscopic, and retroperitoneoscopic approach. J Laparoendosc Adv Surg Tech A 13:159-165

42. Ertem M, Karahasanoglu T, Yavuz N et al (2002) Laparoscopically treated liver hydatid cysts. Arch Surg 137:1170-1173

43. Khoury G, Abiad F, Geagea T et al (2000) Laparoscopic treatment of hydatid cysts of the liver and spleen. Surg Endosc 14:243-245

44. Manterola C, Fernandez O, Munoz S et al (2002) Laparoscopic pericystectomy for liver hydatid cysts. Surg Endosc 16:521-524 